\title{
Fetal demise on MRI
}

\author{
Elspeth H. Whitby
}

Received: 7 September 2011 / Accepted: 5 October 2011 /Published online: 27 October 2011

(C) Springer-Verlag 2011

Sir,

I read with interest the paper by Victoria et al. [1] titled "MR evaluation of fetal demise." Whilst I am pleased to see such work published and the effort the authors have taken with the work, I feel it is worth pointing out to the authors and other readers a few points that need to be considered when interpreting fetal death on MRI.

Several of the image features pointed out by the authors depend on the scanner type and sequence used and care should be taken to avoid overinterpretation.

White blood in the chambers of the heart is frequently seen on balanced gradient echo sequences and must not be mistaken as fetal death.

Skin odema can be seen in fetuses with hydrops and, again, is not diagnostic of fetal demise. In addition, if the fetus died several weeks prior to scanning, there is often no skin odema but a small featureless fetus - the fetal papyrus.

Small pleural effusions are common in cases of fetal demise but again are seen in other pathologies.

E. H. Whitby $(\bowtie)$

Academic Department of Reproductive and Developmental

Medicine, University of Sheffield,

Royal Hallamshire Hospital, Glossop Road,

Sheffield, UK S10 2JF

e-mail: e.whitby@sheffield.ac.uk
The lack of fluid in the stomach and bladder depends on the time between fetal demise and MR imaging and may also be a feature of other pathologies.

The changes in the eyes are not seen after a recent demise and I am unsure as to what time after demise the changes start to occur.

In my experience, the most reliable and consistent feature of fetal demise is the traditional Spalding sign seen on MR images as overlapping of the sutures of the skull and total lack of extra-axial CSF space. Beware of the lack of CSF space in cases with meningoceles; these do not have overlapping sutures.

Loss of grey-white matter differentiation can be due to pathology other than demise, although this often is indicative of a sick fetus where demise is imminent. When seen in association with loss of the extra-axial CSF space, this is probably the best sign of fetal demise of any duration seen on fetal MR images.

As mentioned by the authors, the use of a cine sequence for fetal cardiac motion is useful but ultimately real-time ultrasound is required prior to any change in management.

\section{Reference}

1. Victoria T, Capilla E, Chauvin NA et al (2011) MR evaluation of fetal demise. Pediatr Radiol 41:884-889 